\title{
Aplicación del coeficiente de confiabilidad de Kuder Richardson en una escala para la revisión y prevención de los efectos de las rutinas formadas durante el periodo de confinamiento a partir de la identificación del seguimiento de medidas de seguridad, de comida y de descanso
}

\begin{abstract}
Application of the Kuder Richardson reliability coefficient on a scale for the review and prevention of the effects of the routines formed during the period of confinement from the identification of the monitoring of security measures, food and restain Subtitle in English
\end{abstract}

Fernanda B. Durán-Pérez ${ }^{a}$ Gabriel E. Lara-Abad ${ }^{b}$

\begin{abstract}
:
Reliability is and has undoubtedly been one of the most critical issues for psychology, which, although it has been taking from various knowledge and formal processes from mathematics and statistics everything necessary to be able to develop instruments with a level of approach to current demands, there are often various obstacles. Internal consistency refers to the degree of the project and through which the scale is interrelated with itself. The calculation formula for internal consistency is The Kuder-Richardson formula, from the dichotomous scale or Guttman scalogram, and Cronbach's Alpha coeficient is used for multiple response scales. Consider the internal consistency if it ranges between 0.70 and 0.90 , it is acceptable. Determining the consistency of the scale requires 5 to 20 participants. For each item. The internal consistency of the instrument varies according to the population when applying it, it is always necessary to indicate the value each time a new application is submitted.
\end{abstract}

\section{Keywords:}

Reliability, Scale, Psychometrical assessments

\section{Resumen:}

La confiabilidad es y ha sido sin duda, uno de los temas más álgidos para la psicología, que, si bien ha ido tomando de varios saberes y procesos formales desde la matemática y la estadística todo lo necesario para poder desarrollar instrumentos con un nivel de acercamiento a las demandas actuales, suelen presentarse diversas trabas. La consistencia interna se refiere al grado del proyecto y a través del cuál, la escala está interrelacionada consigo misma. La fórmula de cálculo para la consistencia interna es La fórmula de Kuder-Richardson, de la escala dicotómica o escalograma Guttman y El Alfa de Cronbach se utiliza para escalas de respuestas múltiples. Considerar la consistencia interna si oscila entre 0,70 y 0,90, es aceptable. Determina la consistencia de la escala requiere de 5 a 20 participantes. Para cada artículo. La consistencia interna del instrumento varía según la población al aplicarlo, siempre es necesario indicar el valor cada vez que se presenta una nueva solicitud.

\section{Palabras Clave:}

La Fiabilidad, escala, evaluaciones psicométricas

\section{Introducción}

La investigación científica en cualquier ciencia ha sido de ayuda, no solamente para clasificar como tal sino para que avance en más ámbitos y se vayan descubriendo cosas más importantes sobre la ciencia sobre algún tema en concreto.
Sabemos que cada ciencia ha tenido un inicio complicado, siempre llevado por lo subjetivo. Esto le ha pasado mucho a la psicología, incluso le sigue pasando hoy en día.

Sin embargo gracias a esta investigación científica se han logrado demasiados avances, como la aplicación de la psicología en diferentes ámbitos, así como que a la psicología se le vaya tomando en cuenta para más actividades de la vida diaria. 
Considero que a la psicología ha sufrido demasiadas exclusiones, sin embargo día con día ha demostrado como sustentarse y sobre todo mostrar diferentes aplicaciones considerando el mínimo margen de error como cualquier ciencia aplicada directamente puede tener. Así mismo el papel de la medición en psicología es fundamental para tests que nos servirán para cualquier área laboral que quieras ejercer.

Puede que al principio se pensara que estas mediciones terminarían con toda "humanidad" que existiera, sin embargo en realidad, muestra con la humanidad que se cuenta y con la que no, en bases de datos, y con resultados que ayudaran a más personas a idear nuevos mercados, o maneras de ayudar a más gente vulnerable. La psicología es una ciencia, tan importante como las demás. Para mí, es incluso más importante, porque no solo habla de materia, sino de la mayor población existente en la tierra, de la que dependen más especies y de la que todos somos parte.

La confiabilidad de tipo consistencia interna se refiere al grado en que los ítems, puntos o reactivos que hacen parte de una escala se correlacionan entre ellos, la magnitud en que miden el mismo constructo. Si los puntos que componen una escala teóricamente miden el mismo constructo deben mostrar una alta correlación, es decir, la escala debe mostrar un alto grado de homogeneidad el mismo principio de las fórmulas más tradicionales y conocidas en el contexto de la psicometría. Sin embargo, las más conocidas son la prueba de alfa de Cronbach y la fórmula 20 de Kuder-Richardson.

La fórmula 20 de Kuder-Richardson se indica para el cálculo de la consistencia interna de escalas dicotómicas y el coeficiente de alfa de Cronbach para escalas politómicas.

Estos coeficientes tienen equivalencia matemática. Es importante tener presente que este tipo de coeficientes sólo se puede calcular apropiadamente a escalas que miden atributos o características y no el conocimiento sobre un tópico particular, es decir, no se puede determinar la consistencia interna a una prueba de conocimiento que se aplica en un colegio o universidad, o sea, que necesitan entrenamiento o conocimiento previo en un tópico particular. Aunque, habitualmente se acepta por lo general que la consistencia interna de una escala puede estar en el rango entre 0 y 1 . Lo los valores podrían estar entre -1 y 1 . Valores de 1 e indicarían una correlación entre ítems perfecta, lo cual sería sumamente difícil de lograr en psicología; valores de 0 , ninguna correlación; y coeficiente de -1 , una correlación negativa entre los ítems dónde uno influye de manera anversa el otro.

Es posible ver coeficientes extrañamente negativos cuando se calcula la consistencia interna para una escala que consta de sólo dos ítems. Cómo calcular la consistencia interna. Fórmulas para calcular la consistencia interna de una escala con patrón de respuesta politómica:

Alfa de Cronbach $=\mathrm{k} \mathrm{r} / 1+\mathrm{r}(\mathrm{k}-1)$

$\mathrm{k}$, número de ítems

$\mathrm{r}=\Sigma \mathrm{r} / \mathrm{nr}$

$\mathrm{r}$, correlación de Pearson

$\mathrm{nr}=\mathrm{k}(\mathrm{k}-1) / 2$

La consistencia interna de una escala se considera aceptable cuando se encuentra entre 0,75 y 0,90. Otros sugieren que la consistencia interna de un instrumento es buena si el coeficiente alcanza valores entre 0,80 y 0,90, sobre todo cuando se está iniciando la construcción de la escala.

El error siempre es posible en cualquier medición. Se observa un coeficiente alfa de 0,85 se puede asumir que el 85 de la variabilidad es cierta y el 15 restante probablemente producto del error inherente al tipo de medición, el error no sistemático.

Aplicaciones prácticas de los coeficientes de consistencia interna El proceso de validación de instrumentos es un proceso continuo en constante evaluación que invita a modificaciones que se basen en las investigaciones disponibles. Siempre se necesario hacer adaptaciones lingüísticas cuando se traducen escalas de otros idiomas y, aún, en el mismo idioma cuando el contexto cultural es distinto.

Es importante conocer siempre la consistencia interna de un instrumento en una población específica.

La consistencia interna no es una propiedad básica de una escala, sino la medida del patrón de respuesta del instrumento en el grupo que responde la escala, es decir, que observar un alto valor consistencia interna para una escala en una población, por ejemplo de adultos jóvenes, no garantiza un coeficiente similar en otra con alguna característica sociodemográfica en común, por ejemplo, en adultos mayores.

Igualmente, esto sugiere que los resultados observados pueden indicar la necesidad de adaptaciones de la escala para poblaciones específicas. Aplicaciones prácticas de los coeficientes de consistencia interna 
El proceso de validación de instrumentos es un proceso continuo que obliga a hacer cambios desde las investigaciones previas. Así como hacer traducciones con un buen sistema cuando se pasan escalas de otros idiomas y en el mismo idioma cuando el nivel cultural es distinto.

Es importante conocer siempre la consistencia interna de un instrumento en una población específica. Es frecuente observar estudios en los que se usan escalas y no se informa la consistencia interna de las puntuaciones en la muestra investigada; en cambio, se presenta la consistencia interna que se encontró en otro estudio. Aunque, la consistencia interna es una medida de confiabilidad es un indicador indirecto de la validez en dicha población.

La COVID-19 llego de manera inesperada, sin embargo, primero se debe de aclarar que es. "La COVID-19 es la enfermedad infecciosa causada por el coronavirus que se ha descubierto más recientemente." según La Organización Mundial de la Salud. Tanto este virus como la enfermedad brotaron en Wuhan, China en diciembre del 2019, sin embargo hoy en día la COVID-19 es una pandemia por la cual ya se lleva en confinamiento, hablando exclusivamente de México desde el 11 de Marzo del 2020, aunque algunos establecimientos implementaron esta técnica días después.

La OMS estableció que la propagación de este virus es por medio de gotículas que salen despedidas ya sean de la nariz o boca de una persona ya infectada, pudiendo ser al estornudar, toser, o incluso hablar. Algo importante de recalcar es que esta gotículas viaja al menos a un metro de distancia de los demás, por lo que puede caer sobre diferentes objetos y superficies que la rodean, de modo que si otra persona llega a tocar estos objetos y posteriormente se tocan los ojos, boca u ojos pueden llegar a infectarse. Por ello se han implementado medidas de seguridad para prevenir la propagación de la enfermedad:

- No realizar o asistir a reuniones de más de 50 personas, sin embargo, este acto fue llevado incluso por algunos estados a que se generaran multas y prohibiciones.

- Lavado frecuente de manos así como uso frecuente de un desinfectante a base de alcohol

- Estornudar o toser aplicando la etiqueta de la distancia

- No saludar de beso, ni de mano o abrazar

- Mantener una distancia mínima de un metro de distancia entre persona y persona
- Evitar tocarse los ojos, nariz y boca

- El uso constante de mascarillas o cubre bocas

- Lavar o desinfectar lo que se llegue a comprar, hacer esto después de él lavado de manos

Todas estas medidas de seguridad han llegado a hacer que el ser humano cree rutinas. Según la Real Academia Española rutina es aquella "Costumbre o hábito adquirido de hacer las cosas por mera práctica y de manera más o menos automática." Sin embargo, se ha notado que desde el punto de vista científico las rutinas surgen y se llegan a moldear por que el cerebro busca continuamente minimizar el esfuerzo, en realidad fue demostrado en 2006 por la Universidad de Duke en Estados Unidos que más del $40 \%$ de lo que realiza el ser humano es habitual.

Incluso se ha llegado a comprobar que todos los cambios de conductas que ya son habituales son cambios neurobiológicos.

Es decir, crear rutinas ha ayudado al cerebro humano desde hace años, sin embargo, es importante decir que estas rutinas en cierto modo se han formado e incluso han sido inducidas por varios medios de comunicación.

\section{MÉTODO}

\section{Participantes}

La muestra se integró por 36 sujetos, de los cuales 11 fueron hombres y 25 fueron mujeres. El rango de edad fue de 14 a 35 años, resultando una media de 22 años.

\section{Instrumento}

Se utilizó una escalograma de Guttman, contituida por 47 ítems y 6 factores: Datos generales, Rutina de estudio y trabajo, Cubrebocas, Desinfectar, Comida y descanso, y Percepción.

\section{Procedimiento}

Se elaboró una escala de 47 ítems, posteriormente se aplicó el instrumento por medio de un formulario de google a una muestra de 36 sujetos, buscando la mayor paridad posible de hombres y mujeres. Una vez concluida esta etapa, se procedió a realizar el tratamiento estadístico correspondiente al escalograma de Guttman.

Se desarrolla una matríz para poder generar un análisis efectivo sobre los índices e indicadores y se procede a desarrollar las afirmaciones 
EDAD:

SEXO:

Instrucciones:

A continuación se presentan una serie de afirmaciones en las que te pido puedas elegir de las 2 respuestas que se encuentran debajo de cada una de éstas, aquella que se acerque o sea la que más coincida con tu situación, indicándola con un círculo alrededor de la palabra.

1. Usted vive sólo

2. Tiene familia pero no vive con ella

3. Vive con más de 4 personas

4. En estos momentos tiene trabajo

5. Realiza home office mínimo 3 veces a la semana

6. En estos momentos no estudia

7. Usted realiza la comida del hogar mínimo 3 veces a la semana.

8. Hay cooperación dentro de la casa para la limpieza de ésta .

9. Tiene un horario establecido para el trabajo/ estudio

10. Se para normalmente a diferente hora

11. Alguna vez se le ha hecho tarde para conectarse a clase/junta

12. No ha realizado alguna video llamada en cama

13. No tiene un espacio establecido para el trabajo/estudio

14. Alguna vez ha usado la parte inferior o superior de una pijama o pants en una clase/junta

15. Sufre más de 3 interrupciones a la semana durante una clase/ junta

16. No ha sufrido algún error técnico durante una clase/junta

17. No prefiere este tipo de modalidad

18. No usa cubre bocas cuando llega a salir de casa

19. No usa cubre bocas cuando está dentro de casa

20. Usa cubre bocas para salir a la esquina de su casa

21. Al salir al supermercado o algún centro comercial se quita el cubre bocas

22. Le agrada el uso del cubre bocas

23. Le han salido granos a causa del cubre bocas

24. Llega a sentir que se sofoca mientras usa el cubre bocas

25. Llega a sentir que es necesario el cubre bocas

26. Normalmente no usa el cubre bocas tipo N95

27. Normalmente no usa cubre bocas de tela

28. Usualmente usa gel antibacterial aun cuando no llega a salir

29. Normalmente no llega a usar gel antibacterial después de haber tocado algún objeto ajeno

30. Después de llegar del supermercado o plaza comercial no desinfecta las cosas que compró

31. Después de llegar del supermercado o plaza comercial donde paso más de 1 hora se desinfecta o se baña al llegar a casa

32. Ha sentido humectadas sus manos a causa del gel antibacterial
33. Le agrada el uso del gel antibacterial

34. No tiene un horario establecido de comida

35. No tiene un horario establecido para levantarse de la cama

36. No logra descansar entre semana

37. Logra descansar los fines de semana

38. Antes usted sentía que tenía menos tiempo para descansar entre semana

39. Antes usted sentía que tenía más tiempo para descansar los fines de semana

40. Antes contaba con un horario de comida establecido

41. Antes no tenía un horario establecido para levantarse de la cama

42. Usualmente llega a sentirse fastidiado de permanecer mínimo 3 veces a la semana en casa

43. No ha logrado usted a acostumbrarse a la modalidad en casa

44. Le agrada la modalidad en casa

45. Prefiere la modalidad presencial

46. Llega a sentirse "encerrado"

47. Ha llegado a notar que le guste la modalidad en casa a algún familiar o amigo cercano

\section{Resultados}

Se desarrolla ahora, con los resultados ya trabajados en el programa Excel de la suite de Office de Microsoft y se calcula el coeficiente de reproductibilidad para estar seguros de que se está trabajando adecuadamente el escalograma

\begin{tabular}{|c|c|}
\hline TOTAL DE ERRORES & 20.28 \\
\hline ítems & 47 \\
\hline Sujetos & 36 \\
\hline CR (PASOS) & 1692 \\
\hline & 0.011984502 \\
\hline $\begin{array}{c}\text { CR (COEFICIENTE DE } \\
\text { REPRODUCTIBILIDAD) }\end{array}$ & 0.988015498 \\
\hline CR CON 2 DECIMALES & 0.99 \\
\hline
\end{tabular}

Posteriormente se desarrolla, al tener ya el CR, el índice de reproductibilidad mínima:

\begin{tabular}{|c|c|}
\hline $\begin{array}{l}\text { CR (COEFICIENTE DE } \\
\text { REPRODUCTIBILIDAD) }\end{array}$ & 0.988015498 \\
\hline $\begin{array}{l}\text { CE (COEFICIENTE DE } \\
\text { ESCALABILIDAD) }\end{array}$ & 0.42 \\
\hline & 0.43 \\
\hline
\end{tabular}

Luego, al estar debajo de .60 se entiende que se cuenta con un nivel aceptable.

Así se reordenan a los sujetos para calcular los minimos y máximos de los puntajes obtenidos. 


\section{Re ordenación de sujetos}

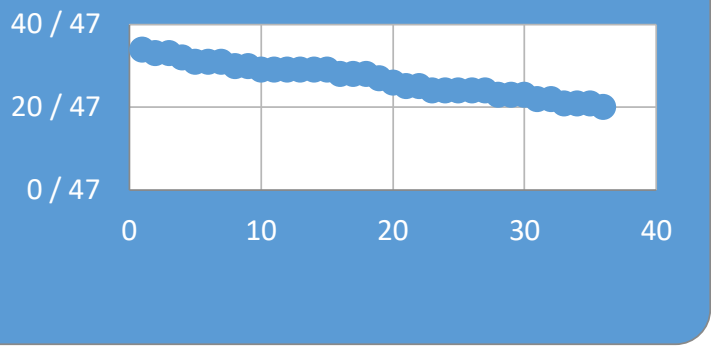

Se obtiene una varianza $\left(\mathrm{S}^{2}\right) 15.58$

Con este dato se procede a obtener la K-de Richardson como previamente se ha explicado.

\begin{tabular}{|l|r|r|r|}
\hline $\begin{array}{l}\text { No Total } \\
\text { items }\end{array}$ & 47 & -1 & 46 \\
\hline & & & \\
\hline & & $\mathrm{Ktt}=$ & 0.39 \\
\hline & & & \\
\hline & $\mathrm{K} / \mathrm{K}-1$ & 1.02173913 & \\
\hline & & 0.38 & \\
\hline
\end{tabular}

\section{Discusión y conclusión}

Se obtiene una $\mathrm{Ktt}=0.39$, lo que nos coloca por debajo de 0.75 , que es el mínimo aceptable. Así se entiende que el nivel obtenido aún no puede llegar a los límites mínimos, esto puede deberse a varios elementos, algunos externos al instrumento como la $\mathrm{N}$ con la que se trabajó, y otros internos como el desarrollo de la organización de los ítems entre sí mismos. El uso del lenguaje o bien, el modelo de organización de los contenidos que quedan en esta visión.

El instrumento tiene congruencia teórica en su construcción, pues los ítems y factores se desarrollaron sobre los tipos de indicadores que son útiles para evaluar. Lo anterior coincide con las afirmaciones realizas y quienes señalan que el constructo está integrado para su evaluación por las dimensiones objetiva y subjetiva e igualmente, se enuncia que la percepción de satisfacción con diferentes factores asociados al desarrollo personal, la salud, el aspecto laboral y las relaciones sociales, entre otros, permite tener datos sobre la calidad de vida de las personas, planteamiento que coincide con el de otras investigaciones.

\section{Referencias}

[1] Arita, B., Romano, S., García, N. y Félix, M. (2005). Indicadores objetivos y subjetivos de la calidad de vida $32-48$.

[2] González, U. (2002). El concepto de calidad de vida y la evolución de los paradigmas de las ciencias de la salud. Revista Cubana

[3] Grau, J. (1999). Calidad de vida y salud: problemas actuales en su investigación. Boletín Latinoamericano de Psicología de la

[4] Lugo A., J., Barroso Á., M. C. y Fernández G., L. (1996). Calidad de vida y sus instrumentos de medición. Una herramienta en

[5] Cañete-Villafranca R, Guilhem D, Brito-Pérez K. Paternalismo médico. Rev. Med. Elect. 2013; 35(2): 144-152.

[6] Trujillo, S., Tovar, C. y Lozano, M. (2004). Formulación de un modelo teórico de la calidad de la vida desde la psicología. Universitas Psicológica, 3, 89-98..

[7] WATSON, Jean. Assessing and Measuring Caring in Nursing and health Science. New York: Springer Publishing Company, 2002. 